\title{
Adaptación y validación de escalas de medición en el trabajo. Parte 2: eficacia colectiva
}

\author{
María L. Campos-Carreño, Camila B. Velasco y Javiera P. Araya \\ Facultad de Humanidades, Departamento de Psicología, Universidad de La Serena, La Serena-Chile \\ (correo-e: mcampos@userena.cl; cvelasco@userena.cl; javiera.arayac@userena.cl)
}

Recibido Abr. 1, 2020; Aceptado May. 28, 2-020; Versión final Jul. 27, 2020, Publicado Dic. 2020

\begin{abstract}
Resumen
El objetivo de este estudio fue adaptar y validar el test de Eficacia Colectiva para Profesores a contextos laborales transversales. La muestra estuvo compuesta por 332 trabajadores pertenecientes a sectores de minería, educación, salud y otros, divididos aleatoriamente en dos submuestras. Se realizaron dos análisis factoriales confirmatorios con el método de estimación de mínimos cuadrados ponderados multivariantes. El primer análisis reveló ajuste adecuado en los parámetros de CFI (comparative fix index) y TLI (Tucker-Lewis index), no así para el RMSEA (root mean square error of approximation). El segundo análisis arrojó un nuevo modelo de medida con ajuste en todos los parámetros de medición (CFI, TLI y RMSEA), constituido por las siguientes dimensiones: competencias grupales positivas y negativas y análisis de elementos positivos y negativos influyentes en las tareas. Se concluye que la escala de Eficacia Colectiva en el Trabajo presenta propiedades psicométricas requeridas para su uso, aportando a la psicología del trabajo y las organizaciones.
\end{abstract}

Palabras clave: eficacia colectiva; competencias grupales; escala Likert; psicología del trabajo

\section{Adaptation and validation of a work measurement scale. Part 2: collective efficacy}

\begin{abstract}
The objective of the present study was to adapt and validate the Collective Teacher Efficacy scale to transversal work contexts. The sample was composed of 332 workers from mining, education, health, and other sectors, randomly divided into two subsamples. Two confirmatory factors were analyzed by using the multivariate weighted least squares analysis method. The first analysis revealed adequate adjustment in the CFI (comparative fix index) and TLI (Tucker-Lewis index) parameters, but not for RMSEA (root mean square error of approximation). The second analysis yielded a new measurement model with adequate adjustment for all parameters (CFI, TLI, and RMSEA) and consisted of the following dimensions: positive and negative group competencies and analysis of positive and negative elements influencing tasks. It is concluded that the Collective Teacher Efficacy scale meets the psychometric properties required for its application in the psychology of work and organizations.
\end{abstract}




\section{INTRODUCCIÓN}

En la actualidad, el aumento de la complejidad del mundo laboral ha cambiado el perfil de competencias que las personas deben desplegar para desempeñarse eficientemente. Una de estas competencias se relaciona con la capacidad para trabajar colaborativa y cooperativamente (Torrelles, Coiduras, Isus, Carrera, Paris y Cela, 2011), dado que el desempeño colectivo y trabajo en equipo constituyen piedras angulares de las organizaciones (Alcover, Rico, y Gil, 2011). Las corrientes actuales plantean la necesidad de hacernos cargo de la eficacia desde una perspectiva colectiva, concibiendo la organización como un sistema social cuyas dinámicas relacionales, cognitivas o socioafectivas tienen un alto impacto en el desempeño personal y grupal, lo que se constituye en un área de estudio creciente (Climént, 2009; Leo,García,Sánchez y Parejo, 2008; Torrelles, et al., 2011).

El concepto de Eficacia Colectiva (EC) surge a partir de los postulados de Albert Bandura en su Teoría Social Cognitiva (TSC) y se define como la percepción de las capacidades ejecutivas de un determinado grupo humano respecto a tareas significativas que le son propias y, en base a su naturaleza social, se requiere activar coordinación efectiva entre sus miembros para realizarlas exitosamente (Volante, 2010; López y Gallegos, 2014; Montilla, 2014). La EC influye en las decisiones que se toman, aspiraciones y expectativas, nivel de esfuerzo, perseverancia, desempeño, resistencia a la adversidad y estrés (Fernández, Diez-Nicolas, Vittorio, Barbaranelli y Bandura, 2004). Bandura (1997) plantea que las creencias de eficacia se adquieren y desarrollan a través de: logros de ejecución o experiencias de dominio, información vicaria, persuación verbal a través de refuerzo social, críticas positivas y palabras de ánimo; y estados fisiológicos o activación emocional.

La EC no está constituida por la suma de eficacias personales (Bandura, 1999), por cuanto su desarrollo requiere disposición personal de los actores sociales para colaborar con otros, intercambiar información, asumir responsabilidad, resolver dificultades que se presenten y contribuir al logro de objetivos (Torrelles, et al., 2011). A su vez, aumenta los niveles de productividad e innovación (Rousseau, Aube y Savoie, 2006; Torrelles, et al., 2011), promueve estados emocionales positivos en los trabajadores, aumento de persistencia frente a los problemas, engagement y el desarrollo de organizaciones saludables que potencian el bienestar colectivo del grupo (Goddard, 2001). Asimismo, supone la presencia de competencias colectivas definidas como las capacidades que se atribuyen a un colectivo que se mantiene en interacción, colaboración constante, coordinación de acciones y logro de resultados comunes (Goddard y Skrla, 2006; De Juanas et al., 2009; Krippendorff, 2009).

Acorde a Goddard, et al. (2000), la EC constituye un factor mediador en el tipo de futuro que los sujetos pueden imaginar, en la forma que se organizan para gestionar sus recursos y en cómo son capaces de elaborar planes y estrategias en conjunto. Aquellos colectivos que sostienen creencias de EC orientan sus metas a afrontar los retos y adversidades que los nuevos contextos les deparan, enfatizando el afrontamiento de errores como una retroalimentación facilitadora de aprendizaje (Tabernero y Wood, 2009). Losada y Heaphy (2004), por su parte, destacan que las relaciones interpersonales positivas dentro de un equipo constituyen un papel determinante en el desempeño de los colectivos, ya que favorecen la conciencia e influencia mutua.

En la actualidad, las investigaciones de EC arrojan que este constructo se relaciona con la autoeficacia requerida para el trabajo en equipo, el alocentrismo horizontal y la percepción de tareas que requieren interdependencia, se vincula significativamente con satisfacción laboral y compromiso organizacional, se asocia positivamente con el logro y favorece una mejor toma de decisiones y desempeño (Caprara, Barbaranelli, Borgogni y Steca, 2003; Volante, 2010; Borgogni, Dello Russo y Latham, 2011; Beckmann, Wood, Minbashian y Tabernero, 2012; Babak y McCormick, 2018).

Para medir este constructo se han construido instrumentos focalizados en áreas de desempeño específicas, como por ejemplo: Scale for use in Public Health and development Programms (Delea, et al., 2018), Collective Efficacy Questionnaire for Sports (Öncü, Feltz, Lirgg y Gürbüz, 2018), Collective Teacher Efficacy (Goddard, Hoy y Woolfolk, 2000), entre otros. No obstante, su mayor cuerpo de investigación se ha generado en el ámbito educacional tanto a nivel nacional como internacional, por lo que resulta imperante la creación y/o adaptación de instrumentos válidos y confiables que permitan medir este constructo en el contexto laboral transversal en Chile. Por tanto, el objetivo de la presente investigación es adaptar y validar a todo contexto laboral la Escala "Collective Teacher Efficacy" de Goddard, Hoy y Woolfolk (2000), creada originalmente para los sistemas educacionales.

\section{MÉTODO}

El diseño de la investigación fue de tipo instrumental de acuerdo a la clasificación de metodologías de investigación para Psicología establecida por Montero y León (2002). 


\section{Participantes}

La muestra fue de tipo no probabilística y estuvo compuesta por 332 sujetos, todos trabajadores activos, mayores de 18 años, con contrato de trabajo superior a tres meses, pertenecientes a los siguientes sectores laborales: minería (supervisores y operadores), educación (directivos y docentes), salud (profesionales y técnicos), servicios administrativos y contables (profesionales y técnicos) y seguridad (guardias). La muestra fue aleatoriamente dividida en dos submuestras. Para la depuración piloto la submuestra A estuvo compuesta por 173 sujetos de los cuales $33.5 \%$ eran hombres $(n=58)$ y $66.5 \%$ mujeres $(n=115)$, con una edad media de 44.02 años (DT=9.8). La muestra para la evaluación final de la escala en estudio (submuestra B) quedó conformada por 159 sujetos, $66.7 \%$ hombres $(n=106)$ y $33.3 \%$ mujeres $(n=53)$, con edad media de 44.18 años (DT=6.2).

\section{Instrumento}

La Escala "Collective Teacher Efficacy" es de tipo Likert, en su versión original posee un Alfa de Cronbach de 0.96 y consta de 21 ítems organizados en las siguientes dimensiones: Análisis positivo o negativo de la tarea ( $A T+$ y $A T$-) definida como la evaluación positiva o negativa que realizan los docentes de los recursos contextuales, humanos, materiales, motivacionales y estructurales que potencialmente favorecen $u$ obstaculizan la realización de una determinada labor; y, Competencias grupales positivas o negativas (CG+ y CG-) entendida como la valoración positiva o negativa que realizan los profesores en cuanto a la disposición, capacidad y convicción de grupo o colectivo docente para desplegar saberes y habilidades favoreciendo $u$ obstaculizando el desempeño de las tareas y logro de metas (Goddard, Hoy y Woolflok, 2000).

\section{Procedimiento}

Para el logro del objetivo de este estudio se procedió a traducir al idioma español y validar el Collective Teacher Efficacy Scale en una muestra de trabajadores chilenos. Se utilizó el proceso de traducción inversa (forward-backward translation) que implica dos fases, la primera conlleva traducir el instrumento de su idioma original al idioma deseado. En una segunda fase, es traducido del idioma deseado al original. De este modo, se cuenta con dos versiones del instrumento en su versión original que de ser idénticas, sugieren que la versión en el idioma deseado es equivalente al original.

En la primera fase, se constituyó un equipo compuesto por un lingüista, un traductor y la investigadora principal, quienes tradujeron de forma individual los reactivos al idioma español (forward translation) generando un documento unificado. La versión traducida al español, se entregó a un segundo equipo integrado por dos psicólogas laborales y un segundo traductor, quienes tradujeron de modo individual la versión en español al idioma inglés (backward translation), generando un nuevo documento unificado. El resultado del proceso se sometió a evaluación por parte de un experto en construcción de instrumentos bilingüe, quien validó la versión traducida del instrumento.

Posteriormente se adaptaron los 21 ítems de la escala "Collective Teacher Efficacy" (referidos exclusivamente al área educacional), a todo contexto laboral (Tabla 1). El proceso de adaptación consideró criterios de aplicabilidad a contextos de trabajo transversales, correspondencia a la dimensión a la que pertenece originalmente cada ítem y ajuste a la intencionalidad semántica de cada reactivo. Los ítems adaptados se presentaron a dos jueces chilenos, psicólogos del trabajo y las organizaciones con amplia trayectoria en recursos humanos, de manera de asegurar que los reactivos fueran comprendidos por los examinados y se ajustarán a los siguientes criterios: equivalencia, comprensión, redacción y habitualidad.

Posterior al proceso de adaptación, se realizó una aplicación piloto de la escala adaptada a la submuestra A con el objetivo de estimar la bondad de ajuste del modelo de 21 ítems mediante análisis factorial confirmatorio. Luego se ejecutó una aplicación de la escala a la submuestra B con el propósito de confirmar el modelo de medida de 21 ítems. Los resultados implicaron un nuevo modelo de medida de 19 ítems, versión depurada de la escala que fue sometida a un nuevo análisis factorial confirmatorio, que estableció las características psicométricas finales de la escala. Junto con lo anterior, con el programa IBM SPPS Statistics V.22 se estableció la consistencia interna para ambas submuestras mediante el coeficiente alfa de Cronbach.

Los análisis factoriales confirmatorios se realizaron con el programa MPLUS V. 7.4, en base al método de estimación mínimos cuadrados ponderados multivariantes. La interpretación de los datos fue realizada considerando los siguientes parámetros: Alfa de Cronbach mayor o igual a 0.75 corresponde a confiabilidad aceptable y mayor o igual 0.90 a confiabilidad elevada (Sampieri, Fernández y Baptista, 2014); Root Mean Square Error of Approximation (RMSEA) menor a 0.05 indica un ajuste adecuado y valores entre 0.05 y 0.08 representan un ajuste razonable (Browne y Cudeck, 1993); Comparative Fix Index (CFI) e Index Tucker-Lewis (TLI) con valores iguales o mayores a 0.95 presentan un buen ajuste (Ruiz, Pardo y San Martín, 2010). 
Tabla 1: Adaptación de los ítems de la escala Collective Teacher Efficacy al Trabajo

\section{Collective Teacher Efficacy (Goddard, Hoy y Woolflok, 2000)}

1. Los profesores en esta escuela tienen lo que se requiere para conseguir que los niños aprendan.

2. Los profesores en esta escuela pueden lograr resultados con estudiantes difíciles.

3. Si un niño no aprende algo la primera vez, los profesores intentarán otras formas de enseñarle.

4. Los profesores aquí tienen la confianza de

\section{Los profesores aquí realmente creen que} todos los niños pueden aprender.

6. Si los alumnos no quieren aprender los profesores aquí se darán por vencidos.

7. Los profesores aquí necesitan más entrenamiento para saber cómo lidiar con estos estudiantes.

8. Los profesores en esta escuela piensan que hay algunos estudiantes a los que no se les puede enseñar.

9. Los profesores aquí no tienen las habilidades necesarias para producir aprendizajes significativos en los estudiantes.

10. Los profesores aquí fallan en enseñar a algunos estudiantes debido a sus pobres métodos de enseñanza.

11. Algunos estudiantes llegan a la escuela listos para aprender.

12. La vida familiar provee muchas ventajas que permiten que los estudiantes aprendan.

13. La falta de recursos y material instruccional hace muy difícil el enseñar.

14. Los estudiantes aquí no están motivados para aprender.

15. La calidad de las instalaciones en este establecimiento realmente facilita el proceso de enseñanza y aprendizaje.

16. Las oportunidades en esta comunidad ayudan a asegurar que estos estudiantes aprenderán.

17. Los profesores aquí están bien preparados para enseñar las asignaturas que se les han asignado.

18. Los profesores de esta escuela son expertos en diversos métodos de enseñanza. que serán capaces de motivar a sus estudiantes.

\section{Dimensión Eficacia Colectiva en el Trabajo}

1. Los trabajadores en esta empresa tienen lo que se requiere para conseguir buenos resultados.

2. Los trabajadores en esta empresa pueden lograr buenos resultados a pesar de situaciones difíciles.

CG+ 3. Si el trabajo no está resultando como se espera los trabajadores intentarán otras formas para mejorarlo.

4. Los trabajadores en esta empresa tienen la confianza de motivar a sus compañeros de trabajo.

5. Los trabajadores en esta empresa realmente creen que todos pueden hacer un buen trabajo.

6. Si los resultados no son óptimos los trabajadores se darán por vencidos.

7. En esta empresa los trabajadores necesitan más entrenamiento para desempeñarse eficazmente.

8. Los trabajadores de esta empresa piensan que hay objetivos laborales que no pueden dominar.

9. Los trabajadores aquí carecen de las habilidades necesarias para lograr resultados favorables.

CG-

10. Aquí los trabajadores tienen débiles métodos de trabajo.

11. Algunos trabajadores llegan a la empresa con disposición para trabajar.

AT+ $\quad$ 12. La vida del hogar de los trabajadores produce ventajas que ayudan a su buen desempeño en el trabajo.

13. La carencia de materiales y recursos hace muy

AT- difícil trabajar.

14. Los trabajadores aquí no están motivados para lograr buenos resultados.

15. La calidad de las instalaciones en esta empresa realmente facilita el trabajo.

AT+

16. En esta empresa existen oportunidades que ayudan a que los trabajadores aprendan y se desempeñen de mejor manera.

17. Los trabajadores están bien preparados para realizar sus funciones y tareas específicas.

$\mathrm{CG}_{+}$

18. Los trabajadores en esta empresa dominan diversas técnicas para el logro de los resultados esperados.

19. Trabajar es más difícil en esta empresa porque los trabajadores están preocupados de su seguridad.

porque los estudiantes están preocupados por su seguridad.

20. El abuso de droga y alcohol en la comunidad hace difícil el aprendizaje para los estudiantes aquí.

21. Los profesores en esta escuela no tienen las habilidades para tratar con estudiantes conflictivos.
AT-

20. El abuso de drogas y alcohol en la empresa dificulta el incremento de los resultados.

21. Los trabajadores en esta empresa no tienen las perjudicar el logro de los resultados. CG- $\quad$ habilidades para manejar los problemas que puedan

Ambas aplicaciones fueron realizadas por un equipo de psicólogos, previamente capacitados, en el lugar de trabajo de los participantes, de manera grupal con administración individual por medio de papel y lápiz, y con 
una duración aproximada de 15 minutos. Todos los participantes accedieron a formar parte de la investigación de manera voluntaria, lo cual se corroboró mediante el consentimiento informado (e.g. participación voluntaria, fines de la investigación, acceso a datos, posibles consecuencias de la aplicación). Asimismo, cada organización firmó los consentimientos correspondientes a nivel institucional.

\section{RESULTADOS}

\section{Análisis factorial Confirmatorio piloto}

El primer paso consistió en verificar la normalidad del conjunto de datos correspondiente a la submuestra A, por medio de la asimetría y la curtosis (Tabla 2).

Tabla 2. Estadísticos Descriptivos Submuestra $A(n=173)$

\begin{tabular}{|l|r|r|r|r|r|}
\cline { 2 - 6 } \multicolumn{1}{c|}{} & \multicolumn{1}{c|}{ CG+ } & \multicolumn{1}{c|}{ CG- } & \multicolumn{1}{c|}{ AT+ } & \multicolumn{1}{c|}{ AT- } & Escala Total \\
\hline Media & 23,494 & 18,335 & 13,129 & 12,200 & 67,159 \\
\hline Error estándar de la media & 0,260 & 0,269 & 0,136 & 0,205 & 0,668 \\
\hline Desviación estándar & 3,390 & 3,501 & 1,773 & 2,668 & 8,706 \\
\hline Varianza & 11,494 & 12,260 & 3,143 & 7,120 & 75,803 \\
\hline Asimetría & $-0,414$ & $-0,472$ & $-0,385$ & $-0,140$ & $-0,057$ \\
\hline Error estándar de asimetría & 0,186 & 0,186 & 0,186 & 0,186 & 0,186 \\
\hline Curtosis & $-0,299$ & 0,394 & 0,465 & $-0,828$ & $-0,754$ \\
\hline Error estándar de curtosis & 0,370 & 0,370 & 0,370 & 0,370 & 0,370 \\
\hline Mínimo & 11,000 & 6,000 & 7,000 & 5,000 & 45,000 \\
\hline Máximo & 28,000 & 24,000 & 16,000 & 16,000 & 83,000 \\
\hline Alfa de Cronbach & 0.829 & 0.669 & 0.539 & 0.514 & 0.960 \\
\hline
\end{tabular}

Considerando la normalidad de los datos obtenidos se procedió con el análisis factorial confirmatorio piloto de la escala adaptada de 21 ítems, cuyo resultado mostró un ajuste adecuado en dos de los parámetros de medición: $\mathrm{CFI}=0.906$ y TLI $=0.893$ no así en RMSEA=0.101. La Tabla 3 muestra las estimaciones de saturaciones factoriales de la escala de 21 ítems, las que exhiben representaciones moderadas $(\lambda \geq 0.30)$ a grandes $(\lambda \geq 0.50)$ de las variables latentes (Cohen, 1988).

Tabla 3. Coeficientes lambdas estandarizados de cada dimensión del modelo de medida

\begin{tabular}{|c|c|c|c|c|c|c|c|}
\hline \multicolumn{2}{|l|}{$C G_{+}$} & \multicolumn{2}{|l|}{ CG- } & \multicolumn{2}{|l|}{$A T+$} & \multicolumn{2}{|l|}{$A T-$} \\
\hline Ítem & $\Lambda$ & Ítem & $\Lambda$ & Ítem & $\Lambda$ & Ítem & $\Lambda$ \\
\hline 1 & 0.623 & 6 & 0.674 & 11 & 0.763 & 13 & 0.674 \\
\hline 2 & 0.631 & 7 & 0.537 & 12 & 0.440 & 14 & 0.793 \\
\hline 3 & 0.735 & 8 & 0.775 & 15 & 0.287 & 19 & 0.512 \\
\hline 4 & 0.908 & 9 & 0.654 & 16 & 0.715 & 20 & 0.491 \\
\hline 5 & 0.861 & 10 & 0.874 & & & & \\
\hline 17 & 0.839 & 21 & 0.809 & & & & \\
\hline 18 & 0.842 & & & & & & \\
\hline
\end{tabular}




\section{Análisis factorial confirmatorio}

Posteriormente, por medio de la asimetría y la curtosis se verificó la normalidad del conjunto de datos correspondiente a la submuestra B (Tabla 4).

Tabla 4: Estadísticos Descriptivos Submuestra B ( $n=159)$

\begin{tabular}{|l|c|c|c|c|c|}
\cline { 2 - 6 } \multicolumn{1}{c|}{} & CG+ & CG- & AT+ & AT- & Escala Total \\
\hline Media & 23,033 & 17,994 & 12,981 & 11,864 & 65,870 \\
\hline Error estándar de la media & 0,278 & 0,279 & 0,155 & 0,213 & 0,694 \\
\hline Desviación estándar & 3,450 & 3,465 & 1,928 & 2,645 & 8,618 \\
\hline Varianza & 11,901 & 12,006 & 3,719 & 6,994 & 74,271 \\
\hline Asimetría & $-0,294$ & $-0,469$ & $-0,543$ & $-0,001$ & 0,038 \\
\hline Error estándar de asimetría & 0,195 & 0,195 & 0,195 & 0,195 & 0,195 \\
\hline Curtosis & $-0,357$ & 0,361 & 0,884 & $-0,823$ & $-0,773$ \\
\hline Error estándar de curtosis & 0,389 & 0,389 & 0,389 & 0,389 & 0,389 \\
\hline Mínimo & 11,000 & 6,000 & 6,000 & 5,000 & 45,000 \\
\hline Máximo & 28,000 & 24,000 & 16,000 & 16,000 & 83,000 \\
\hline Alfa de Cronbah & 0.836 & 0.781 & 0.590 & 0.632 & 0.860 \\
\hline
\end{tabular}

Con el objetivo de medir la estabilidad factorial del modelo de 21 ítems en la submuestra B se realizó un nuevo análisis factorial confirmatorio. Acorde a los criterios de ajuste más comunes en la literatura especializada, el modelo en análisis no constituye una explicación suficiente de la matriz de covariaciones observada. Por ello, se procedió a revisar la estructura factorial inicial lo que resultó en la extracción de dos ítems (no 2 y no 14) en base a los siguientes criterios: elementos redundantes y baja saturación factorial. Para favorecer el ajuste de este nuevo modelo, con 19 ítems, se aplicó la Varianza común del método. El nuevo modelo obtiene mejores indicadores de ajuste acorde a lo señalado por diversos autores (Tabla 5).

Tabla 5: Indicadores de Ajuste Escala de Eficacia Colectiva en el Trabajo

\begin{tabular}{|l|l|l|l|}
\hline Criterios de ajuste & $\begin{array}{l}\text { RMSEA } \\
0.05 \text { y } 0.08\end{array}$ & $\begin{array}{l}\text { CFI } \\
\geq 0.95\end{array}$ & $\begin{array}{l}\text { TLI } \\
\geq 0.95\end{array}$ \\
\hline Modelo con 21 ítems & 0.105 & 0.889 & 0.873 \\
\hline Modelo con 19 ítems & 0.075 & 0.948 & 0.938 \\
\hline
\end{tabular}

La Figura 1 presenta gráficamente la versión final de la Escala de Eficacia Colectiva en el Trabajo y especifica las estimaciones de saturaciones factoriales, categorizadas en representaciones moderadas $(\lambda \geq 0.30)$ a grandes $(\lambda \geq 0.50)$ de las variables latentes (Cohen, 1988). Se Confirman las 4 dimensiones establecidas por Goddard, Hoy y Woolfolk (2000) Competencias Grupales Positivas (CG+) Competencias Grupales Negativas (CG-) Análisis de elementos positivos influyentes en las tareas (AT+) Análisis de elementos negativos influyentes en las tareas (AT-).

La escala final de Eficacia Colectiva en el Trabajo en formato Likert, cuenta con 4 categorías de respuesta (totalmente en desacuerdo, en desacuerdo, de acuerdo, totalmente de acuerdo), calificados de 1 (totalmente en desacuerdo) a 4 (Totalmente de acuerdo). La puntuación total indica el nivel de Eficacia Colectiva en base a un sistema de clasificación, cuya suma por dimensiones permite obtener un registro ordinal. Para establecer las puntuaciones de la escala se utilizó puntuaciones típicas o estándar lineales directas. Estas calificaciones señalan la distancia, en términos de desviaciones típicas, en que se encuentra un puntaje original con respecto a la media de su distribución de puntajes originales. 
En la submuestra $B$ la media correspondió a $(X=58.76)$ y desviación Estándar $(\sigma=8.57)$. En esta muestra los trabajadores que alcanzan puntajes inferiores a 50.19 poseen Baja Eficacia Colectiva; entre 67.33 y 50.19 corresponden a Eficacia Colectiva Media y puntajes superiores a 67.33 califican Alta Eficacia Colectiva.

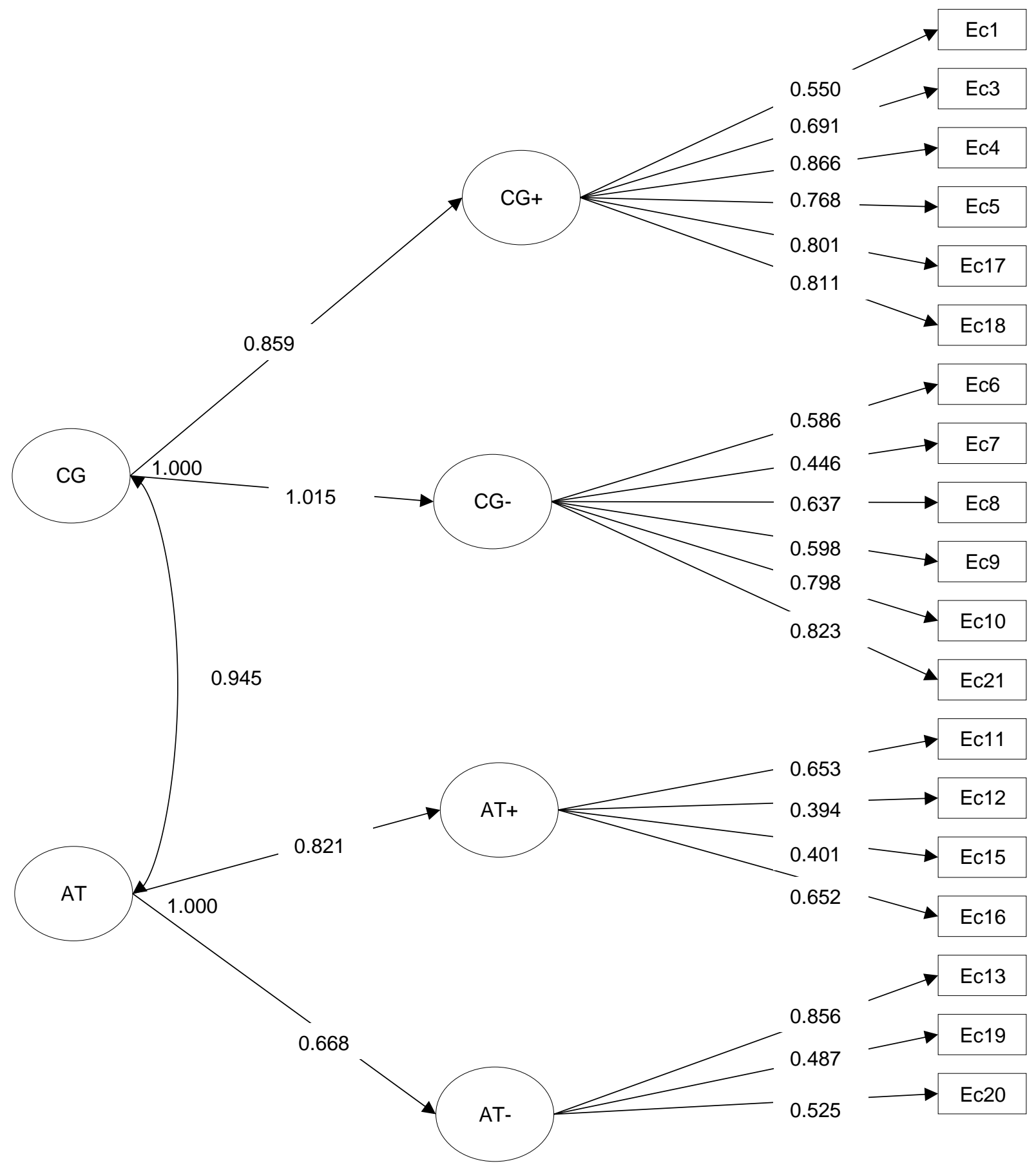

Fig. 1: Representación Gráfica del Modelo de Eficacia Colectiva en el Trabajo

\section{DISCUSIÓN}

Se define Eficacia Colectiva en el Trabajo como la valoración de las capacidades ejecutivas y de la existencia de recursos con que cuenta un determinado colectivo o sistema social respecto a tareas significativas que le son propias y en las que se requiere activa coordinación efectiva entre sus miembros. La Escala propuesta, otorga valor a las dimensiones de Competencias Grupales; lo que confirma lo planteado por diversos autores, quienes al referirse a la Eficacia Colectiva suponen incremento de competencias colectivas positivas (Rousseau, Aube y Savoie, 2006; Torrelles, et al., 2011). Tal como señalan De Juanas et al., (2009) y Krippendorf (2009), las competencias colectivas positivas son capacidades que se atribuyen a un colectivo, que se mantiene en interacción y colaboración constante, coordinando acciones y llegando a resultados comunes. 
La versión final de la escala (Tabla 6) confirma las 4 dimensiones planteadas por Goddard, Hoy y Woolfolk (2000) siendo redefinidas acorde a la transversalidad de contextos laborales que aborda: 1)Competencias Grupales Positivas $\left(\mathrm{CG}_{+}\right.$) valoración positiva que realizan los trabajadores en cuanto a la disposición, capacidad y convicción del grupo o colectivo para desplegar saberes, habilidades, actitudes e intercambios, en base a una participación activa y combinada de esfuerzos con la finalidad de producir un resultado común y co-construir soluciones; 2) Competencias Grupales Negativas (CG-) valoración negativa que realizan los trabajadores en cuanto a la existencia de disposición, o capacidad del grupo o colectivo para desplegar saberes, habilidades, actitudes e intercambios, lo que obstaculiza el logro de resultados y limita la participación activa en resultados compartidos; 3)Análisis de elementos positivos influyentes en las tareas $(A T+)$ entendido como la evaluación positiva que realizan los trabajadores de los recursos contextuales, humanos, materiales, motivacionales y estructurales que potencialmente favorecen la realización de una determinada labor; y 4)Análisis de elementos negativos influyentes en las tareas (AT-) evaluación negativa ejecutada por los trabajadores de los recursos contextuales, humanos, materiales, motivacionales y estructurales lo que dificulta u obstaculiza la realización de una labor.

Tabla 6: Escala de Eficacia Colectiva en el Trabajo

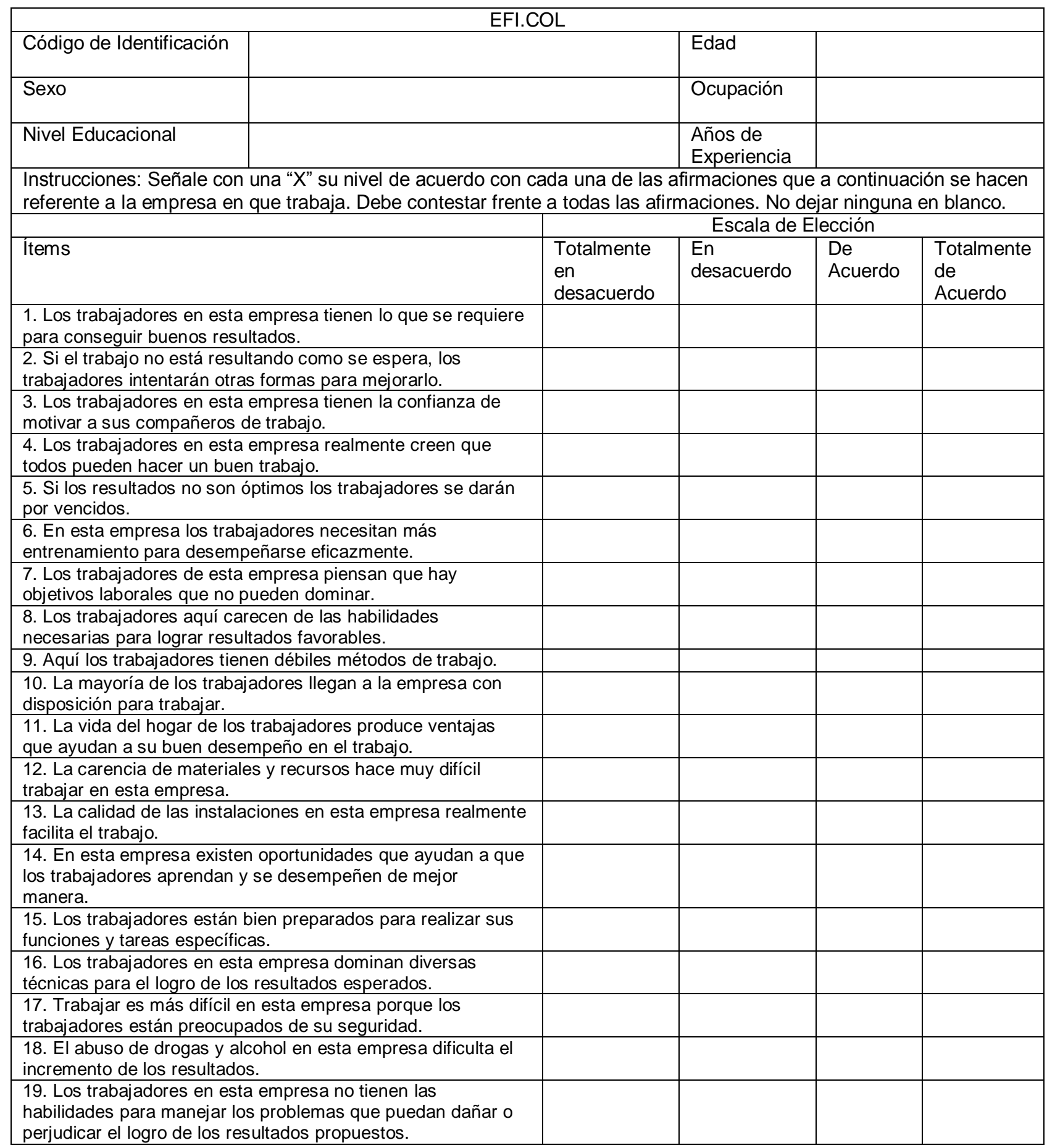


La Escala obtenida valora los elementos influyentes en el desempeño laboral, lo que corrobora lo planteado por Engeström (2008), quien considera que en un sistema de actividad son relevantes las competencias de los participantes, tanto como los elementos influyentes en el desempeño laboral. Este autor plantea que las competencias de cooperación, coordinación y comunicación son fundamentales en el proceso de funcionamiento y desarrollo de los colectivos, otorgando valor a la construcción de vínculos, nudo que sostiene a quienes participan juntos. Pero también hace referencia a la transformación expansiva como un viaje colectivo a través de la zona de desarrollo próximo de la actividad; que incluye factores protectores y factores de riesgo, lo que repercute en la generación de perturbaciones/ rupturas, y expansiones. Lo cual queda corroborado en esta investigación por cuanto los elementos positivos influyentes en las tareas favorecen las expansiones; mientras que los elementos negativos influyentes en las tareas estimulan las perturbaciones y rupturas, impactando negativamente la eficacia colectiva (Engeström, 2008).

\section{CONCLUSIONES}

El propósito de este estudio fue adaptar y validar la escala "Collective Teacher Efficacy" de Goddard, Hoy y Woolfolk (2000) creada originalmente para los sistemas educacionales a contextos laborales transversales. Los resultados expuestos permiten afirmar que la escala de "Eficacia Colectiva en el Trabajo" propuesta constituye un instrumento válido y confiable acorde a estándares vigentes, constituyendo un aporte a las demandas diagnósticas de la Psicología del Trabajo y las Organizaciones, tanto a nivel de investigación como de acción/intervención, aplicable a cualquier ámbito de empleo.

\section{REFERENCIAS}

Alcover, C.M., Rico, R., y Gil, F., Equipos de Trabajo en Contextos Organizacionales; Dinámicas de Cambio, Adaptación y Aprendizaje en Entornos Flexibles, Papeles del Psicólogo, ISSN: 0214 - 7823, 32(1), 7-16 (2011)

Babak, S. y McCormick, J. Why do I think my team is capable? A study of some antecedents of team members' personal collective efficacy beliefs, doi: 10.1080/01443410.2018.1500680, Educational Psychology, 38(9), 1147-1162 (2018)

Bandura, A., Self-efficacy: The Exercise of Control, 1 . Ed., W.H. Freeman,79-115, New York, Estados Unidos (1997)

Bandura, A. Auto-eficacia: Cómo Afrontamos los Cambios de la Sociedad Actual, $1^{\circ}$ Ed., Descleé de Brower S.A., 1-280, Bilbao, España (1999)

Beckmann, N., Wood, R.E., Minbashian, A., y Tabernero, C., Small Group Learning: Do Group Members's Implicit Theories of Ability Make a Difference? doi: https://doi.org/10.1016/j.lindif.2012.06.007, Learning and Individual Differences,22(5), 624-631(2012)

Borgogni, L., Dello Russo, S. y Latham, G., The Relationship of Employee Perceptions of the Immediate Supervisor and Top Management with Collective Efficacy, doi: 10.1177/1548051810379799, Journal of Leadership \& Organizational Studies, 18(1), 5-13 (2011)

Browne, M. W. y Cudeck, R., Alternative Ways of Assessing Model Fit, Bollen, K. y Long J. (Ed.), Testing structural equation models, 136-162, Sage, California, Estados Unidos (1993)

Caprara, G. V., Barbaranelli, C., Borgogni, L. y Steca, P., Efficacy Beliefs as Determinants of Teachers' Job Satisfaction, doi: 10.1037/0022-0663.95.4.821, Journal of Educational Psychology, 95(4), 821-832 (2003)

Climént, J. B., El Papel de las Competencias Individuales y Colectivas en los Sistemas de Acción, Actualidades Investigativas en Educación, ISSN: 1409-4703, 9(2), 1-19 (2009)

Cohen, J. Statistical power analysis for the behavioral science, $2^{\circ}$ Ed, Lawrence Erlbaum Associates, Publishers, 467-514559, New Jersey, Estados Unidos (1988)

De Juanas, A., Fernández, P. y otros cuatro autores, Comparative study of the evaluation of professional competencies by experienced and trainee Spanish primary teachers, doi: 10.1080/02619760903005823, 32(4), European Journal of Teacher Education, 437-454 (2009)

Delea, M. G., Sclar, G. D., y otros ocho autores, Collective Efficacy: Development and Validation of a Measurement Scale for Use in Public Health and Development Programmes, doi: 10.3390/ijerph15102139, International Journal of Environmental Reaserch and Public Health, 15, 1-38 (2018)

Engeström, Y., From teams to knots: activity-theoretical studies of collaboration and learning at work., $1^{\circ}$ Ed., 48-63, Cambridge University Press, Nueva York, Estados Unidos (2008)

Fernández, B., Diez-Nicolás, y otros tres autores, Determinantes y relaciones estructurales desde la eficacia personal a la eficacia colectiva, Salanova, M., Grau, R. M., Martínez, I. M., Cifre, E., Llorens, S. y García-Renedo, M. (Eds.), Nuevos horizontes en la investigación sobre la autoeficacia, 69-80, Publicacions de la Universitat Jaume, España (2004)

Goddard, R. D., Collective Efficacy: A Neglected Construct in the Study of Schools and Student Achievement, doi: https://doi.org/10.1037/0022-0663.93.3.467, Journal of Educational Psychology, 93, 467-476 (2001) 
Goddard, R. D., y Skrla, L., The influence of School social composition on teachers' collective efficacy beliefs. doi: https://doi.org/10.1177/0013161X05285984, Educational Administration Quarterly, 42(2), 216-235 (2006)

Goddard, R. D., Hoy, W. K., y Woolfolk, A., Collective Teacher Efficacy: Its meaning, measure, and impact on student achievement, doi: 10.3102/00028312037002479, American Educational Research Journal, 37(2), 479 - 507 (2000)

Krippendorf, K., Ross Ashby's information theory: a bit of history, some solutions to problems and what we face today, doi: 10.1080/03081070802621846, International Journal of General Systems, 38(2), 189-212 (2009)

Leo, F. M., García, T., Sánchez , P. A., y Parejo, I., Importancia de la Percepción de Eficacia para la Mejora de la Cohesión en el Fútbol, Cuadernos de Psicología del Deporte, ISSN: 1989-5879, 8(1), 47-60 (2008)

López, P., y Gallegos, V., Prácticas de Liderazgo y el Rol Mediador de la Eficacia Colectiva en la Satisfacción Laboral de los Docentes, Estudios pedagógicos, ISSN: 0718-0705, 40(1), 163-178 (2014)

Losada, M. y Heaphy, E., The Role of Positivity and Connectivity in the Performance of Business Teams: a Nonlinear Dynamics Model, doi: 10.1177/0002764203260208, American Behavioral Scientist, 47(6), 740-765 (2004)

Montero, I., y León, O. G., Clasificación y Descripción de las Metodologías de Investigación en Psicología, Revista Internacional de Psicología Clínica y de la Salud, ISSN: 2174-0852, 2(3), 503-508, (2002)

Montilla, S., Trabajo, Rendimiento y Bienestar: Una Propuesta Actual de Organización Positiva, Licenciado, Universidad de Jaen (2014)

Öncü, E., Feltz, D. L., Lirgg, C. D., y Gürbüz, B., The Examination of the Psychometric Properties of the Turkish Collective Efficacy Questionnaire for Sports, doi: 10.5507/ag.2018.005, Acta Gymnica, 48(1), 27-35 (2018)

Rousseau, V., Aube, C. y Savoie, A., Teamwork Behaviors a Review and an Integration of Frameworks, doi: 10.1177/1046496406293125, Small Group Research, 37(5), 540-570 (2006)

Ruiz, M. A., Pardo, A. y Martín, R., Modelo de Ecuaciones Estructurales, Papeles del Psicólogo, ISSN: 0214 - 7823, 31(1), 35-45 (2010)

Sampieri, R, Fernández, C., y Baptista, P., Metodología de la Investigación, 5². ed., 276-343, McGraw Hill, D.F., México (2014)

Tabernero, C. y Wood, R. E., Interaction Between Self-Efficacy and Initial Performance in Predecting the Complexity of Task Chosen, doi: https://doi.org10.2466/PR0.105.F1167/1180, Psychological Reports, 105(3), 1167-1180 (2009)

Torrelles, C., Coiduras, J., y otros cuatro autores, Competencias de Trabajo en Equipo: Definición y Categorización, Revista de Currículum y Formación del Profesorado, ISSN 1989-6395, 15(3), 329-344 (2011)

Volante, P. Influencia Instruccional de la Dirección Escolar en los Logros Académicos, Doctor, Pontificia Universidad Católica de Chile (2010) 